\title{
Guillain-Barré syndrome following bacterial meningitis: a case report and literature review
}

\author{
Li Ding $^{1 \dagger}$, Zhongjun Chen ${ }^{2 \dagger}$, Yan Sun ${ }^{3 \dagger}$, Haiping Bao ${ }^{4}$, Xiao Wu' ${ }^{1}$, Lele Zhong ${ }^{1}$, Pei Zhang ${ }^{1}$, Yongzhong Lin ${ }^{1 *}$
} and Ying Liu ${ }^{1 *}$ D

\begin{abstract}
Background: We reported a case of an adult that presented Guillain-Barré syndrome (GBS) after bacterial meningitis which was secondary to chronic suppurative otitis media (CSOM). To our knowledge, this is the first case involving an adult presenting with GBS following bacterial meningitis.

Case presentation: A 46-year man with type 2 diabetes and otitis media (OM) suffered with fever, headache, and vomiting for 6 days. The patient's neck stiffness was obvious and the Kernig and Brudzinski signs were produced. The result of cerebrospinal fluid (CSF) analysis and cytological examination of the CSF supported the diagnose of bacterial meningitis. On day 17 the patient felt numbness in both hands and feet, which gradually progressed to weakness of the limbs. Bladder dysfunction occurred, which required catheterization. The patient showed a tetraparesis with emphasis on the legs. The deep tendon reflexes of limbs were absent. The patient had peripheral hypalgesia and deep sensory dysfunction. The symptoms were possibly a result of GBS. Nerve conduction study showed that the F wave latency of the upper and lower limbs was prolonged, particularly the lower limbs. 8 days later the repeated nerve conduction study showed a low compound muscle action potential $(3.3 \mathrm{mV})$ with a normal distal motor latency $(14.2 \mathrm{~ms})$ and a low motor nerve conduction velocity $(34.3 \mathrm{~m} / \mathrm{s})$ in the tibial nerve. The patient still required assistance when walking 3 months after onset.

Conclusions: GBS following bacterial meningitis is rare and limbs weakness in patients with bacterial meningitis was usually considered because of weakness. This case should serve as a reminder for clinical doctors that when a patient with bacterial meningitis complains about limbs numbness or weakness, GBS should be considered, especially when the patient had diabetes mellitus (DM) history.
\end{abstract}

Keywords: Guillain-Barré syndrome, Bacterial meningitis, Chronic suppurative otitis media

\section{Background}

Guillain-Barré syndrome (GBS) is an acute onset immunemediated disorder of the peripheral nervous system. Patients with this disease usually manifest acute progressive weakness, hyporeflexia or areflexia, and elevated levels of protein in the CSF. Approximately two thirds of patients with GBS experienced a preceding infection or an antecedent event (ie. surgery or immunizations) a few weeks

\footnotetext{
*Correspondence: lin19671024@163.com; 179140376@qq.com

${ }^{\dagger}$ Li Ding, Zhongjun Chen and Yan Sun contributed equally to this work.

'Department of Neurology, the Second Hospital of Dalian Medical University,

No. 467 Zhongshan Road, Shahekou District, Dalian City 116027, Liaoning

Province, China

Full list of author information is available at the end of the article
}

prior to neuropathy [1]. There are only two case reports where Guillain-Barre syndrome appeared following bacterial meningitis. One case is an 11-year-old boy who experienced Guillain-Barre syndrome following meningococcal meningitis [1] and the other case is a previously healthy 35 month old girl who had Listeria meningitis with selective spinal grey matter involvement and exhibited demyelination in the neurophysiological studies [2]. To our knowledge, this is the first case involving an adult presenting with GBS following bacterial meningitis.

(C) The Author(s). 2018 Open Access This article is distributed under the terms of the Creative Commons Attribution 4.0 International License (http://creativecommons.org/licenses/by/4.0/), which permits unrestricted use, distribution, and reproduction in any medium, provided you give appropriate credit to the original author(s) and the source, provide a link to the Creative Commons license, and indicate if changes were made. The Creative Commons Public Domain Dedication waiver (http://creativecommons.org/publicdomain/zero/1.0/) applies to the data made available in this article, unless otherwise stated. 


\section{Case presentation}

A 46-year man with type 2 diabetes (6 years) and OM (a few years) suffered with fever, headache, and vomiting for 6 days. There was purulent blood from the patient's right ear. Magnetic resonance imaging (MRI) showed turbid sulcus (Fig. 1a) and OM of the left ear (Fig. 1. arrows in b). The lower class hospital treated with Cefperazone-Sulbactam for 3 days. The symptoms did not improve so the patient was transferred to the Second Hospital of Dalian Medical University (Dalian, China). The patient's neck stiffness was obvious and the Kernig and Brudzinski signs were produced. The results of blood tests were showed in Table 1. In addition the glycosylated hemoglobin was $11.20 \%$. The patient was diagnosed with diabetic ketoacidosis (DKA) and fluid replacement therapy was implemented. Lumbar puncture was operated on day 7 and the results of CSF analysis were showed in Table 2. The patient was diagnosed bacterial meningitis and treated with Ceftriaxone Sodium $3.0 \mathrm{~g}$ daily and vacocin vancomycin $1.0 \mathrm{~g}$ twice daily. Mannitol was used to dehydrate the cranial pressure. An otoscopy showed a bulging hyperemic tympanic membrane in the left ear and there were some bloody matter on the surface. The external auditory canal was swollen. The patient was diagnosed CSOM by an otolaryngologist and levofloxacin hydrochloride ear drops and erdosteine were prescribed. The lumbar puncture was repeated on day 14 and the results were showed in Table 2. Human albumin was added to dehydrate the cranial pressure. The blood culture was negative after cultured for 5 days. In order to reduce inflammatory reaction, dexamethasone $10 \mathrm{mg}$ daily was added. On day 17 the patient felt numbness in both hands and feet, which gradually progressed to both legs and left arm. Bladder dysfunction occurred, which required catheterization. The patient showed a tetraparesis. Muscle strength in the right arm was grade 4 proximally and grade $4^{-}$distally, in the left arm was grade 3 proximally and grade 2 distally, in the right leg was grade 2 proximally and grade $2^{-}$distally, in the left leg was grade 0 both proximally and distally. The deep tendon reflexes of limbs were absent. The patient had distally pronounced hypalgesia and deep sensory dysfunction in arms and legs. Deep sensory dysfunction reached above wrists and was from the feet up to the knees. The symptoms were possibly a result of GBS. The blood culture was negative after an additional 5 days of culturing. IVIg was initiated at day 20. The patient's symptoms improved, but his neck stiffness remained. The lumbar puncture was repeated on day 21 and the results were showed in Table 2 . On day 22 the nerve conduction study showed that the F wave latency of the upper and lower limbs was prolonged, particularly the lower limbs (Table 3 ). On day 30 the repeated nerve conduction study showed a low compound muscle action potential $(3.3 \mathrm{mV})$ with a normal distal motor latency $(14.2 \mathrm{~ms})$ and a low motor nerve conduction velocity $(34.3 \mathrm{~m} / \mathrm{s})$ in the tibial nerve. The F wave latency of the lower limbs was prolonged (Table 4). Both nerve conduction studies supported the diagnosis of GBS. On day 33 acoustic impedance tests showed hearing loss in the left ear. On day 35 muscle strength in the right arm was grade 5 proximally and grade 5 distally, in the left arm was grade 4 proximally and grade 3 distally, in the right leg was grade 5 proximally and grade 5 distally, in the left leg was grade 4 proximally and grade 3 distally. Deep tendon reflexes were produced in the limbs. He had peripheral deep sensory dysfunction in the lower limbs. The patient was transferred to the local rehabilitation hospital. The patient still required assistance when walking 3 months after onset.

\section{Discussion and conclusions}

GBS is a group of neuropathic diseases. Within 4 weeks of onset there are two-thirds of adult patients who have gastrointestinal tract or respiratory infection [3]. Campylobacter jejuni is found in $25-50 \%$ of the adult patients, and more frequently in Asian countries [4]. One

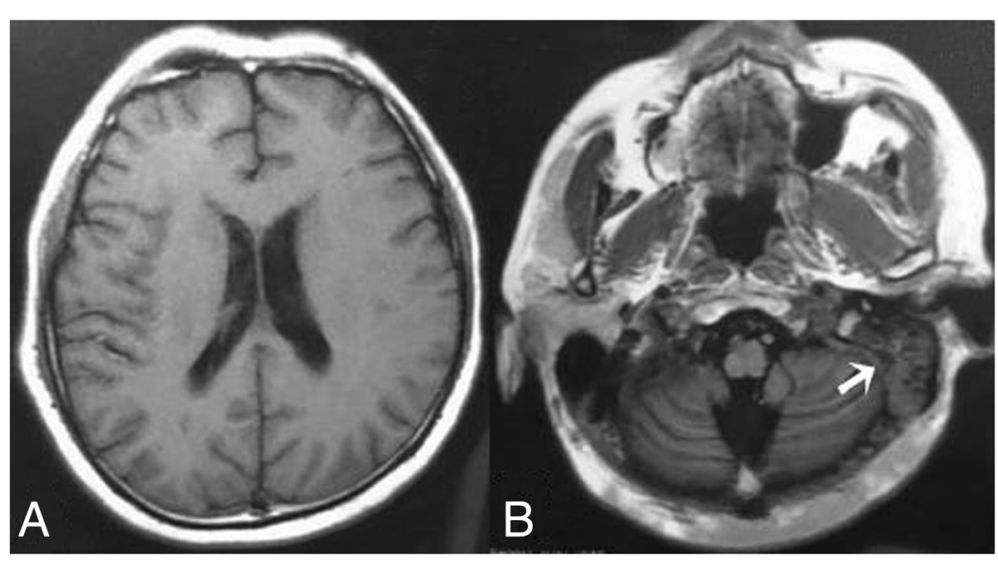

Fig. $1 \mathrm{MRI}$ at the junior hospital showed turbid sulcus (a) and OM in the left ear (arrows in $\mathbf{b}$ ) 
Table 1 The results of blood tests

\begin{tabular}{|c|c|c|c|c|c|c|c|c|}
\hline Characteristics & Reference range & Day 6 & Day 7 & Day 8 & Day 14 & Day 16 & Day 23 & Day 28 \\
\hline$\overline{W B C}$ & $\times 10^{9} / L$ & 23.95 & 21.90 & 12.55 & 11.25 & 10.54 & 6.92 & \\
\hline NEUT\% & $40.00-75.00$ & 90.3 & 89.30 & 82.50 & 75.30 & 90.30 & 72.80 & \\
\hline Fasting blood-glucose & 3.9-6.1 mmol/L & 16.39 & refuse & 17.1 & 14.3 & 7.0 & 11.8 & 8.7 \\
\hline $\mathrm{Cl}^{-}$ & $98.00-107.00 \mathrm{mmol} / \mathrm{L}$ & 112.00 & 106.46 & 101.66 & 95.40 & 98.89 & 92.08 & 96.68 \\
\hline Urinary ketone body & negative & +++ & ++ & negative & negative & negative & & \\
\hline $\mathrm{pH}$ & $7.35-7.45$ & 7.28 & 7.44 & 7.41 & & 7.47 & & \\
\hline $\mathrm{PCO}_{2}$ & $35-48 \mathrm{mmHg}$ & 15.90 & 27.20 & 25.00 & & 34.60 & & \\
\hline SBE & $-1.5-3.0 \mathrm{mmol} / \mathrm{L}$ & -19.50 & -5.10 & -8.80 & & 1.40 & & \\
\hline $\mathrm{CHCO}_{3}^{-}$ & $22.5-26.9 \mathrm{mmol} / \mathrm{L}$ & -11.80 & 20.50 & 18.30 & & 25.70 & & \\
\hline
\end{tabular}

Abbreviations: WBC white blood cell, NEUT neutrophil, $\mathrm{PCO}_{2}$ partial pressure of carbon dioxide, $S B E$ standard base excess, $\mathrm{CHCO}_{3}{ }^{-}$actual bicarbonate

study showed that Mycoplasma pneumoniae was found in $15 \%$ patients of GBS and was the second most common causative agent $[5,6]$. Other causative agents included cytomegalovirus (CMV), Epstein-Barr virus [7], influenza A virus, Listeria monocytogenes [8], Haemophilus influenza [3], hepatitis E [9, 10], Zika and chikungunya [11]. A case report showed a case of Listeria meningitis in a previously healthy 35 month-old female with demyelination in the neurophysiological studies [2]. We reported a case of an adult that presented GBS after bacterial meningitis. Although the CSF was cultured four times and blood cultured twice, the results were all negative. The bacteria that caused this rare symptom remained unknown. The patient's bacterial meningitis was secondary to CSOM. Causative agents of chronic CSOM mainly included Proteus mirabilis, Pseudomonas aeruginosa, Staphylococcus aureus, or anaerobic bacteria [12]. Bacterial meningitis is the most common intracranial complication of both acute and chronic OM. The common organisms caused this complication are $\mathrm{H}$. influenzae Type B, S. pneumoniae, and Group A streptococcus [13]. It is likely that the organism that caused the symptoms in the patient were one of these.
Acute inflammatory demyelinating polyneuropathy and acute motor axonal neuropathy are the main phenotypes of GBS spectrum. The electrophysiological results of our patient revealed a demyelinating neuropathy with reduced motor nerve conduction velocity and prolonged F wave latency, which is in line with an acute inflammatory demyelinating polyneuropathy. The patient presented GBS symptoms on day 17 following bacterial meningitis. Critical illness polyneuropathy (CIP), which manifests as distally predominant limb weakness and reduced reflexes, should be distinguished. CIP is a sensory-motor axonal polyneuropathy that commonly develops in critically ill patients [14]. The electrophysiological characteristics of this neuropathy are reduced amplitude of the compound motor and the sensory nerve action potential, the near normal motor and sensory conduction velocities, the normal F-wave latency and denervation patterns on needle electromyograms [15]. The patient was critically ill but did not meet the criteria that were required for CIP. Thus, this patient appears unique in having GBS following bacterial meningitis.

An adult that presents with bacterial meningitis and GBS is not common. A history of diabetes mellitus (DM)

Table 2 The results of analysis of CSF samples obtained by lumbar puncture

\begin{tabular}{llllll}
\hline Characteristics & Reference range & Day 7 & Day 14 & Day 21 & Day 28 \\
\hline Appearance & & hemorrhagic cloudy & hazy yellow & yellow transparent & yellow transparent \\
Opening pressure (lateral decubitus) & $80-180 \mathrm{mmH}_{2} \mathrm{O}$ & 300 & $>330$ & 265 & 250 \\
WBC count & $0-8 / \mu \mathrm{L}$ & 6400 & 320 & 92 & 11 \\
$\mathrm{~L}$ & $\%$ & 5.00 & 19.00 & 46.00 & 64.00 \\
$\mathrm{~N}$ & $\%$ & 95.00 & 81.00 & 8.20 & 5.30 \\
Glucose & $2.2-3.9 \mathrm{mmol} / \mathrm{L}$ & 8.30 & 9857.70 & 5900.50 & 4545.50 \\
Total protein & $120-600 \mathrm{mg} / \mathrm{L}$ & $13,685.20$ & 107.80 & 112.39 & 117.42 \\
Chlorides & $120-132 \mathrm{mmol} / \mathrm{L}$ & 119.15 & negative & negative & negative \\
CSF cultures & negative & negative & &
\end{tabular}


Table 3 The results of nerve conduction study on day 22 after the onset

\begin{tabular}{|c|c|c|c|c|c|c|}
\hline Nerve & Stimulation & Latency (ms) & Amp. & Velocity $(\mathrm{m} / \mathrm{s})$ & F-mean-latency & $\mathrm{F}-\mathrm{NO} \%$ \\
\hline Motor & & & $(\mathrm{mV})$ & & & \\
\hline \multirow[t]{2}{*}{ Rt. peroneal } & Ankle & $3.5(<3.9)$ & $6.2(>2.0)$ & & & \\
\hline & Fibula (head) & & 5.1 & $40.2(>40.0)$ & & \\
\hline \multirow[t]{2}{*}{ Lt. peroneal } & Ankle & $3.2(<3.9)$ & $8.1(>2.0)$ & & & \\
\hline & Fibula (head) & & 7.0 & $41.5(>40.0)$ & & \\
\hline \multirow[t]{2}{*}{ Rt. tibial } & Ankle & $5.2(<5.1)$ & $8.0(>4.0)$ & & $58.3(<53.9)$ & $100.0(>70.0)$ \\
\hline & Popliteal Fossa & & 8.0 & $43.8(>40.0)$ & & \\
\hline \multirow[t]{2}{*}{ Lt. tibial } & Ankle & $4.3(<5.1)$ & $5.1(>4.0)$ & & $59.9(<53.9)$ & $100.0(>70.0)$ \\
\hline & Popliteal Fossa & & 3.1 & $41.4(>40.0)$ & & \\
\hline \multirow[t]{2}{*}{ Lt. median } & Wrist & $3.3(<4.0)$ & $20.4(>7.0)$ & & $30.9(<29.37)$ & $100.0(>80.0)$ \\
\hline & Elbow & & 20.1 & $51.0(>50.0)$ & & \\
\hline \multirow[t]{2}{*}{ Lt. ulnar } & Wrist & $2.4(<3.1)$ & $16.0(>7.0)$ & & $29.6(<29.1)$ & $100.0(>80.0)$ \\
\hline & Elbow & & 15.6 & $55.5(>50.0)$ & & \\
\hline Sensory & & & $(\mu \mathrm{V})$ & & & \\
\hline Rt. superficial peroneal & Lower leg & 2.2 & $11.7(>5.0)$ & $45.4(>40.0)$ & & \\
\hline \multirow[t]{2}{*}{ Lt. median } & Digit I & 2.5 & $28.6(>15.0)$ & $52.0(>43.4)$ & & \\
\hline & Digit III & 3.0 & $21.9(>6.5)$ & $46.6(>46.5)$ & & \\
\hline Lt. ulnar & Digit V & 1.9 & $9.8(>7.0)$ & $55.2(>45.0)$ & & \\
\hline Lt. radial & Digit I & 1.8 & $26.8(>5.0)$ & $52.7(>40.0)$ & & \\
\hline
\end{tabular}

Abbreviations: Amp amplitude, $L t$ left, $R t$ right, F-NO.\% F wave occurrence rate

could be critical, particularly since the patient's blood glucose was not well controlled. Both DM type 1 and type 2 are associated with increased infection rates, particularly bacterial infections [16]. Infections in patients with diabetes are more likely to be severe [17]. This is likely the reason for the patient's bacterial meningitis being so severe. A recent study which involved 85 patients that had acute polyradiculoneuropathy showed that DM type 2 was present in $32 \%$ patients. The three-month prognosis was worse in patients with both GBS and diabetes [18]. This could be explained in many ways. Some patients with DM may have pre-existing nerve injuries, and the onset of the

Table 4 The results of nerve conduction study on day 30 after the onset

\begin{tabular}{|c|c|c|c|c|c|c|}
\hline Nerve & Stimulation & Latency (ms) & Amp. & Velocity $(\mathrm{m} / \mathrm{s})$ & F-mean-latency & F-NO.\% \\
\hline Motor & & & $(\mathrm{mV})$ & & & \\
\hline \multirow[t]{2}{*}{ Lt. peroneal } & Ankle & $3.6(<3.9)$ & $7.8(>2.0)$ & & $59.5(<54.1)$ & $56.3(>70.0)$ \\
\hline & Fibula (head) & & 7.0 & $40.9(>40.0)$ & & \\
\hline \multirow[t]{2}{*}{ Lt. tibial } & Ankle & $4.0(<5.1)$ & $4.6(>4.0)$ & & $57.6(<53.9)$ & $100.0(>70.0)$ \\
\hline & Popliteal Fossa & & 3.3 & $34.3(>40.0)$ & & \\
\hline \multirow[t]{2}{*}{ Lt. median } & Wrist & $3.3(<4.0)$ & $15.5(>7.0)$ & & $28.7(<29.37)$ & $95.0(>80.0)$ \\
\hline & Elbow & & 14.6 & $51.0(>50.0)$ & & \\
\hline \multirow[t]{2}{*}{ Lt. ulnar } & Wrist & $2.4(<3.1)$ & $14.8(>7.0)$ & & $27.9(<29.1)$ & $100.0(>80.0)$ \\
\hline & Elbow & & 14.7 & $58.3(>50.0)$ & & \\
\hline Sensory & & & $(\mu \mathrm{V})$ & & & \\
\hline Lt. superficial peroneal & Lower leg & 2.6 & $14.6(>5.0)$ & $46.1(>40.0)$ & & \\
\hline Lt. sural & Ankle & 2.7 & $29.0(>5.0)$ & $45.1(>42.0)$ & & \\
\hline \multirow[t]{2}{*}{ Lt. median } & Digit I & 2.5 & $32.9(>15.0)$ & $44.0(>43.4)$ & & \\
\hline & Digit III & 2.6 & $27.5(>6.5)$ & $50.0(>46.5)$ & & \\
\hline Lt. ulnar & Digit $\mathrm{V}$ & 1.9 & $20.6(>7.0)$ & $52.6(>45.0)$ & & \\
\hline
\end{tabular}


GBS worsened the situation [18]. Diabetic patients have reduced rates of nerve regeneration, even before signs and symptoms of neuropathy appear. Once diabetic neuropathy appears, the capacity for nerve regeneration further decreases [19]. Diabetes could also increase the inflammation in GBS, since both diseases are associated with systemic inflammation [18].

In conclusion, GBS following bacterial meningitis is rare. However, our case shows that it should be considered in patients with bacterial meningitis suffering limb weakness and numbness, especially when the patient has DM history. Delayed diagnosis and treatment may result in poor neurological outcomes. This case should serve as a reminder for clinical doctors that when a patient with bacterial meningitis complains about limbs numbness or weakness, GBS should be considered, especially when the patient had DM history. IVIg should be started immediately after the diagnosis to prevent symptom progression.

\section{Abbreviations}

CIP: Critical illness polyneuropathy; CMV: Cytomegalovirus; CSF: Cerebrospinal fluid; CSOM: Chronic suppurative otitis media; DKA: Diabetic ketoacidosis; DM: Diabetes mellitus; GBS: Guillain-Barré syndrome; LOS: Lipooligosaccharides; Lt: Left; MRI: Magnetic resonance imaging; OM: Otitis media; Rt: Right

\section{Acknowledgements}

None.

\section{Funding}

Dr. Ying Liu was supported by Dalian Medical Science Research Project (No. 1712037). Dr. Yongzhong Lin was supported by Chinese National Natural Science Foundation (No. 81571237) and Liaoning Province Science and Technology Project (No. 2017225070). These funding were used in the design of the study and collection, analysis, and interpretation of data and in writing the manuscript.

\section{Availability of data and materials}

The datasets used and/or analysed during the current study are available from the corresponding author on reasonable request.

\section{Authors' contributions \\ All authors took part in the work and agree with the contents of the manuscript $Y L$ and $Y Z L$ provided concepts. HB conducted the nerve conduction study and analyzed the data. LD, ZC, and YS acquired the data. XW, LZ, and PZ did the literature review. YL drafted and submitted the initial manuscript. All authors critiqued and revised the manuscript critically for content. All authors read and approved the final manuscript.}

\section{Ethics approval and consent to participate}

Informed consent was obtained from the patient and this case report was approved by the ethics committee of the Second Hospital of Dalian Medical University.

\section{Consent for publication}

The patient can't write because of the disease. Written informed consent was obtained from the patient's daughter for publication of this case report. The patient also gave verbal consent for publication.

\section{Competing interests}

The authors declare that they have no competing interests.

\section{Author details}

'Department of Neurology, the Second Hospital of Dalian Medical University, No. 467 Zhongshan Road, Shahekou District, Dalian City 116027, Liaoning Province, China. ${ }^{2}$ Neuro-Interventional Ward, Dalian Municipal Central Hospital of Dalian Medical University, Dalian City, China. ${ }^{3}$ Anesthesiology Department, Jilin University, China Japan Union Hospital, Changchun City, China. ${ }^{4}$ Department of Nerve Electrophysiology, the Second Hospital of Dalian Medical University, Dalian City, China.

Received: 29 May 2018 Accepted: 30 November 2018

Published online: 17 December 2018

\section{References}

1. Puri V, Khalil A, Suri V. Guillain-Barre syndrome following meningococcal meningitis. Postgrad Med J. 1995;71:42-3.

2. Papandreou A, Hedrera-Fernandez A, Kaliakatsos M, Chong WK, Bhate S. An unusual presentation of paediatric listeria meningitis with selective spinal grey matter involvement and acute demyelinating polyneuropathy. Eur J Paediatr Neurol. 2016;20:196-9.

3. Jacobs BC, Rothbarth PH, van der Meche FG, Herbrink P, Schmitz PI, de Klerk $M A$, et al. The spectrum of antecedent infections in guillain-Barre syndrome: a case-control study. Neurology. 1998:51:1110-5

4. Islam Z, Jacobs BC, van Belkum A, Mohammad QD, Islam MB, Herbrink P, et al. Axonal variant of guillain-Barre syndrome associated with campylobacter infection in Bangladesh. Neurology. 2010;74:581-7.

5. Sinha S, Prasad KN, Jain D, Pandey CM, Jha S, Pradhan S. Preceding infections and anti-ganglioside antibodies in patients with guillain-Barre syndrome: a single Centre prospective case-control study. Clin Microbiol Infect. 2007;13:334-7.

6. Sharma MB, Chaudhry R, Tabassum I, Ahmed NH, Sahu JK, Dhawan B, et al. The presence of mycoplasma pneumoniae infection and gm1 ganglioside antibodies in guillain-Barre syndrome. J Infect Dev Ctries. 2011;5:459-64.

7. Khatib HE, Naous A, Ghanem S, Dbaibo G, Rajab M. Case report: GuillainBarre syndrome with pneumococcus - a new association in pediatrics. IDCases. 2018:11:36-8.

8. Vergori A, Masi G, Donati D, Ginanneschi F, Annunziata P, Cerase A, et al. Listeria meningoencephalitis and anti-gq1b antibody syndrome. Infection. 2016;44:543-6.

9. Geurtsvankessel CH, Islam Z, Mohammad QD, Jacobs BC, Endtz HP, Osterhaus AD. Hepatitis e and guillain-Barre syndrome. Clin Infect Dis. 2013; 57:1369-70.

10. van den Berg B, van der Eijk AA, Pas SD, Hunter JG, Madden RG, Tio-Gillen $A P$, et al. Guillain-Barre syndrome associated with preceding hepatitis e virus infection. Neurology. 2014;82:491-7.

11. Willison HJ, Jacobs BC, van Doorn PA. Guillain-barre syndrome. Lancet. 2016; 388:717-27.

12. Mittal R, Lisi CV, Gerring R, Mittal J, Mathee K, Narasimhan G, et al. Current concepts in the pathogenesis and treatment of chronic suppurative otitis media. J Med Microbiol. 2015;64:1103-16.

13. Osma U, Cureoglu S, Hosoglu S. The complications of chronic otitis media: report of 93 cases. J Laryngol Otol. 2000;114:97-100.

14. Bird SJ, Rich MM. Critical illness myopathy and polyneuropathy. Curr Neurol Neurosci Rep. 2002;2:527-33.

15. Bolton CF, Laverty DA, Brown JD, Witt NJ, Hahn AF, Sibbald WJ. Critically ill polyneuropathy: electrophysiological studies and differentiation from guillain-Barre syndrome. J Neurol Neurosurg Psychiatry. 1986;49:563-73.

16. Shah BR, Hux JE. Quantifying the risk of infectious diseases for people with diabetes. Diabetes Care. 2003;26:510-3.

17. Calvet HM, Yoshikawa TT. Infections in diabetes. Infect Dis Clin N Am. 2001; 15:407-21 viii.

18. Bae JS, Kim YJ, Kim JK. Diabetes mellitus exacerbates the clinical and electrophysiological features of guillain-Barre syndrome. Eur J Neurol. 2016; 23:439-46.

19. Polydefkis M, Hauer P, Sheth S, Sirdofsky M, Griffin JW, MCArthur JC. The time course of epidermal nerve fibre regeneration: studies in normal controls and in people with diabetes, with and without neuropathy. Brain 2004;127:1606-15

\section{Publisher's Note}

Springer Nature remains neutral with regard to jurisdictional claims in published maps and institutional affiliations. 